\title{
EPSL
}

Earth and Planetary Science Letters 130 (1995) 75-85

\section{Inner core anisotropy, anomalies in the time-averaged paleomagnetic field, and polarity transition paths}

\author{
Bradford M. Clement ${ }^{a}$, Lars Stixrude ${ }^{b, 1}$ \\ ${ }^{a}$ Department of Geology, Florida International University, Miami, FL 33199, USA \\ ${ }^{\mathrm{b}}$ Institut für Geophysik, Herzberger Landstrasse 180, Universität Göttingen, 37075 Göttingen, Germany
}

Received 29 July 1994; accepted after revision 20 December 1994

\begin{abstract}
The diffusion of the dynamo-generated magnetic field into the electrically conducting inner core of the Earth may provide an explanation for several problematic aspects of long-term geomagnetic field behavior. We present a simple model which illustrates how an induced magnetization in the inner core which changes on diffusive timescales can provide a biasing field which could produce the observed anomalies in the time-averaged field and polarity reversals. The Earth's inner core exhibits an anisotropy in seismic velocities which can be explained by a preferred orientation of a polycrystalline aggregate of hexagonal close-packed (hcp) iron, an elastically anisotropic phase. Room temperature analogs of hcp iron also exhibit a strong anisotropy of magnetic susceptibility, ranging from 15 to $40 \%$ anisotropy. At inner core conditions the magnetic susceptibility of hcp iron is estimated to be between $10^{-4}$ and $10^{-3}$ SI. We speculate here that the anisotropy in magnetic susceptibility in the inner core could produce the observed anomalies in the time-averaged paleomagnetic field, polarity asymmetry, and recurring transitional virtual geomagnetic pole (VGP) positions.
\end{abstract}

\section{Introduction}

When averaged over sufficiently long periods the Earth's magnetic field very closely approximates that of a geocentric axial dipole. Numerous studies of the time-averaged paleomagnetic field, however, have documented small but consistent deviations from a purely axial, geocentric dipole field [1-13]. These studies consistently observe equatorial inclinations which are too steep com-

\footnotetext{
${ }^{1}$ Currently on leave from: School of Earth and Atmospheric Sciences, Georgia Institute of Technology, Atlanta, GA 30332-0340, USA

pared with those expected from a true axial dipole field. These steep inclinations give rise to the so-called far-sided effect, in which the paleomagnetic poles fall on the far side of the north geographic pole from the site [2]. This effect has been generally modeled by including a significant axial quadrupole term which is between 2 and $6 \%$ of the axial dipole term in the expression of the time-averaged field. The offset dipole hypothesis, in which the axial dipole is displaced northward along the spin axis, was put forward as a possible explanation of this effect. However, no physical explanation exists for why the dipole should be offset.

Many studies of the time-averaged field have also documented a polarity asymmetry in which 
normal and reverse polarity directions are not perfectly antipodal [2-13]. Although the magnitude of this effect varies from study to study, it appears that the quadrupole term is generally twice as great during intervals of reverse polarity ( $5 \%$ of the dipole term) as it is during periods of normal polarity ( $3 \%$ of the dipole term) [9]. These asymmetric properties of the field are difficult to explain using dynamo theory simply because of the symmetry in the induction equations [4-6]. Therefore the asymmetry must result from boundary conditions on the geodynamo.

Although less well documented than the anomalies in the time-averaged paleomagnetic field, recent studies of polarity transitions suggest that recurrent patterns exist in transitional VGP paths [14-16]. Although much of the discussion of reversal paths has centered on the nearly antipodal paths along the Americas and Asia which occur in several reversals, it is clear that not all reversals exhibit VGP paths which fall along these trends [17,18]. Even in reversals which do not exhibit VGPs falling along these preferred paths, recurrent patterns are often observed within the VGP paths of individual reversals, such as in the Cobb Mountain reversals [16] or the Liverpool volcanics records [19]. Because the dynamo should not possess a memory on these timescales $\left(10^{3}-\right.$ $10^{6} \mathrm{yr}$ ), more persistent boundary conditions have been invoked to explain these recurrent features.

The lowermost mantle is often thought to be the source of these persistent boundary conditions because the timescales of change in mantle conditions are those of mantle convection. Therefore the boundary is permanent relative to the more rapid changes in the fluid outer core. There also exists evidence from seismic tomography that lateral heterogeneities exist in the lowermost mantle [20]. These lateral heterogeneities may be associated with variations in temperature or electrical conductivity which are capable of influencing the geodynamo. While the apparent correlation of fast seismic velocities in the lowermost mantle with the VGP paths over the Americas and Asia suggests that there may be a relation between the two, no mechanism has successfully explained how the lowermost mantle influences the field on global scales.
Although it has received less attention, the inner boundary of the outer core is expected to have a long-term influence on the geomagnetic field as well. The finite electrical conductivity of the solid inner core allows field lines to diffuse into this region and imposes a relatively long, diffusive timescale on the variation in the geomagnetic field. Hollerbach and Jones [21,22] have recently shown that the inner core can have a major influence on the geometry and stability of the geomagnetic field. The magnetic diffusivity of the inner core tends to dampen out large fluctuations and to stabilize the field against reversals.

It is natural to ask how this picture would change if the inner core were anisotropic in its electromagnetic material properties. Seismology has revealed that the inner core is elastically anisotropic, and it may be anisotropic in its electrical conductivity and magnetic susceptibility as well. Certain types of anisotropy are capable of breaking the symmetry of the geodynamo equations and may provide a simple explanation of non-dipolar features of the field geometry such as polarity asymmetry, inclination anomalies and preferred VGP paths.

Here we consider the effects of an anisotropic magnetic susceptibility of the inner core. For realistic susceptibility tensors and crystallographic textures, we find that the induced magnetization of the inner core is (locally) misaligned with the main field. Because of the long diffusive timescale of the inner core, this induced magnetization changes much more slowly than the main field and can produce long-term, non-dipolar features in the main field. We first review the evidence for inner core anisotropy and estimate its intrinsic susceptibility. We combine these estimates with a simple textural model of the inner core and compare its predictions with observed inclination anomalies, the polarity asymmetry and polarity transition records.

\section{The inner core}

A number of studies of seismic wave propagation through the inner core have concluded that the average P-wave velocity along the Earth's 
rotation axis is about $3 \%$ faster than in the equatorial plane (Fig. 1a)[23-25]. These studies, together with free oscillation studies [26,27], conclude that the inner core behaves as an anisotropic medium. The symmetry of the anisotropy is identical to that of a hexagonal single crystal. We assume that the inner core anisotropy is caused by the texturing of an aggregate of hexagonal close-packed ( $\mathrm{hcp}$ ) iron crystals. This explanation, which was originally proposed by Morelli et al. [23], has recently received quantitative support from a forward model of inner core elasticity based on theoretical determination of the elastic constants of hcp iron [28]. The forward modeling indicates that the texture in the inner core is strong, near $100 \%$ alignment.

The physical process responsible for the texturing of the inner core is unknown. Jeanloz and Wenk [29] proposed that it originates from a strain field induced by convection of the inner core. Karato [30] proposed that the texturing results from the interaction of the geomagnetic field with induced fields in iron as it crystallizes in the presence of the field. Uncertainties in the texturing process mean that the geometry of the crystallographic preferred orientation is also unknown. For definiteness we assume the texture predicted by Wenk et al. [31] for a convecting inner core on the basis of shear deformation experiments on metals at ambicnt conditions. The geometry of the lowest order convective mode and the resulting crystallographic preferred orientation is illustrated in Fig. $1 \mathrm{~b}$.

Given our present level of understanding, the assumed texture must be considered as only an illustrative example. Our results depend critically on only one feature of the texture predicted by Wenk et al. [31], namely that there is a substantial component of $c$-axis alignment normal to the spin axis. This is because the magnetic susceptibility of hcp iron is expected to be a second rank tensor, so that its principle components and the direction of the induced field coincide with crystallographic axes. The component of $c$-axis alignment normal to the spin axis allows the induced field to be misaligned with the main field. It is possible that there are other texturing mechanisms and other texture geometries which also

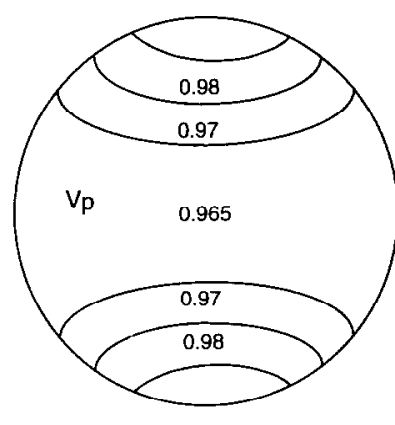

a

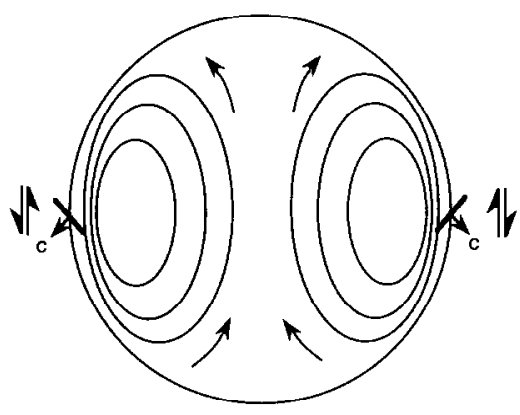

b

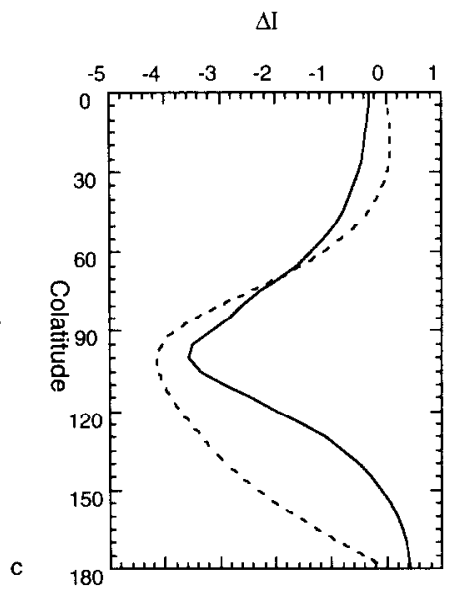

Fig. 1. The inner core is elastically anisotropic, with P-wave velocities slightly faster along the north-south axis (a). Jeanloz and Wenk [29] suggested that the elastic anisotropy may be caused by a preferred orientation of a polycrystalline aggregate of hcp iron which arises from shear strain induced during convection of the inner core. The most fundamental mode of convection produces an alignment of the $c$-axes of hcp iron shown in (b). Hcp iron also likely exhibits an anisotropy of magnetic susceptibility suggesting that a preferred alignment would produce an easy direction(s) of magnetization. An induced dipole $\left(m=10^{-2} M_{\mathrm{E}}\right)$ placed.parallel to the orientation of the $c$-axis at the equator produces an inclination anomaly $(c)(\Delta I$, defined as the observed inclination minus that predicted by a geocentric axial dipole field) at the surface which is in good agreement with the best-fit curve to the inclination anomaly in the time-averaged field. The best-fit curve to the reverse polarity dataset of Schneider and Kent [10] is shown as a dashed line. This geometry demonstrates that an induced field from the inner core of sufficient magnitude could produce the reversing quadrupole field in the time-averaged field studies. 
produce a component of $c$-axis alignment normal to the spin axis and which could also account for the non-dipolar features of the field examined here.

The induced magnetization of the inner core has generally not been considered in dynamo theories because core temperatures are well above the Curie temperature of body centered cubic (bcc) iron at ambient pressure [32]. In ferromagnets, the susceptibility follows a $1 / T$ relation above the Curie temperature and would be expected to be small at inner core temperatures. However, iron is not expected to be ferromagnetic at high pressure. First-principles bandstructure calculations show that at high pressure iron is paramagnetic even at zero temperature. It possesses no local moments [33,34]. Local magnetic moments vanish with increasing pressure because they tend to expand the lattice, which is energetically unfavorable at high pressure.

In contrast to ferromagnets, paramagnetic metals are observed to have susceptibilities which depend only weakly on temperature. This is well understood in terms of the Pauli spin susceptibility of the conduction electrons [35]. These spins obey much more restrictive statistics than those associated with localized moments. Because of the exclusion principle, in a free electron gas only those electrons which are less than $k T$ below the Fermi energy (where $k T$ is the thermal energy) can change their spin in response to an applied magnetic field. The Pauli spin susceptibility $(\chi)$ is to a first order independent of temperature: $\chi \sim$ $1 / T\left(T / T_{\mathrm{F}}\right)=1 / T_{\mathrm{F}}$, where $T_{\mathrm{F}}$ is the Fermi temperature. Comparison of theoretical and experimental results indicate that the Pauli spin susceptibility contributes approximately $40 \%$ of the total susceptibility [36]. The weak temperature dependence of this term is important because it means that we can use relatively low temperature $(T<$ $2000 \mathrm{~K})$ experimental observations of analog compounds to guide our estimate of the likely susceptibility of iron in the inner core. Room temperature values of susceptibility for hcp transition metals range from $10^{-4}$ to $10^{-3} \mathrm{SI}$. The low end of this range may be an underestimate as the closest analogs of $\mathrm{Fe}$ ( $\mathrm{Ti}, \mathrm{Zr}$ and $\mathrm{Hf}$ [28]) all show values of susceptibility which increase steadily with temperature, at least up to $1600 \mathrm{~K}$ $[35,37]$. We estimate a susceptibility of hcp iron of $10^{-3}-10^{-4}$ SI.

Given a paramagnetic susceptibility of this level it is possible that the inner core is significantly magnetized by the fields produced by dynamo activity in the outer core. Although the actual field strength in the outer core is unknown, Zhang et al. [38] have recently put an upper value of on the strength of the toroidal field (B) within the core of $5 \times 10^{-3} \mathrm{~T}$. The poloidal field at the pole is at least $10^{-4} \mathrm{~T}$ and may be more at the base of the outer core. If we assume that in the inner core the inducing field $\mathbf{H}$ is equivalent to the strength of the field in the outer core, we obtain a value of $50 \times 10^{3} / 4 \pi \mathrm{A} / \mathrm{m}$ for the inducing field strength. Using this as an upper limit for the strength of the inducing field and a paramagnetic susceptibility of $10^{-3}$ SI for hcp iron we obtain a dipole moment for a uniformly magnetized inner core of radius $1200 \mathrm{~km}$ of $2.9 \times 10^{19} \mathrm{Am}^{2}$, which is only three orders of magnitude less than the dipole moment of the dynamo produced field. Given the uncertainties in the values of field strengths and susceptibilities we do not place too much confidence in these values. We simply point them out here to make the point that if the susceptibility of hcp iron is close to that estimated based on room temperature analogs, the induced magnetization of the inner core may be significant. However, using the upper bounds on possible fields and susceptibilities we obtain an induced dipole moment of the inner core which may be capable of explaining a number of features of the Earth's magnetic field. As we discuss below, in order for these effects to be observable at the surface they must be transmitted and amplified by the dynamo action occurring in the overlying, conducting outer core.

\section{The reversing quadrupole field}

If the inner core were isotropic, the induced field would on average be aligned with the Earth's spin axis because we know that the dynamo field is axial when averaged over sufficiently long time intervals. If the magnetic susceptibility of the inner core, however, is anisotropic, it is possible 
that the induced field would not be exactly parallel to the inducing field. A preferred alignment of a polycrystalline aggregate of hcp iron in the inner core would likely give rise to an easy direction or directions of magnetization.

Following the geometry of Hollerbach and Jones [21] we assume an axial poloidal field diffuses into the inner core. If we assume that the crystal alignments are similar to those suggested to have resulted from convection in the inner core [29] we find that the induced magnetization in the inner core produces a field geometry which can explain the reversing quadrupole term in the time-averaged paleomagnetic field. The alignment of $c$-axes at $45^{\circ}$ to the horizontal at the equator at the top of the inner core provides the geometry which can produce an equatorial inclination anomaly at the Earth's surface. Placing a dipole moment aligned parallel to the $c$-axis orientation at the equator with a moment which is $10^{-2} \times$ the dipole moment of the dynamo produced field yields $\Delta I$ values close to those observed. The resulting plot of $\Delta I$ with colatitude is remarkably similar to the $\Delta I$ curve obtained by Schneider and Kent [11] which provides the best fit with the time-averaged data from deep-sea cores (Fig. 1c). Changing the polarity of the dynamo field changes the sign of the induced magnetization, and therefore the $\Delta I$ would be symmetric with respect to polarity.

One of the major difficulties in modeling the quadrupolar portion of the standing field has been that there is no strong evidence for a nonzonal component in the time-averaged field. Therefore, any non-zonal field, such as an equatorial dipole, used to produce the quadrupole term must average out to zero with time while not averaging out the axial quadrupole component. For this reason the axisymmetry of Jeanloz and Wenk's [29] grain alignment geometry is important to this model in that an axial, poloidal, inducing field will produce an induced magnetization in the inner core which has a vertical component at the equator along all longitudes. In this way an axisymmetric quadrupole field is produced.

The magnitude of the induced dipole moment which provides a fit to the observed $\Delta I$ anomaly is an order of magnitude greater than that calculated for an inner core with our estimate of
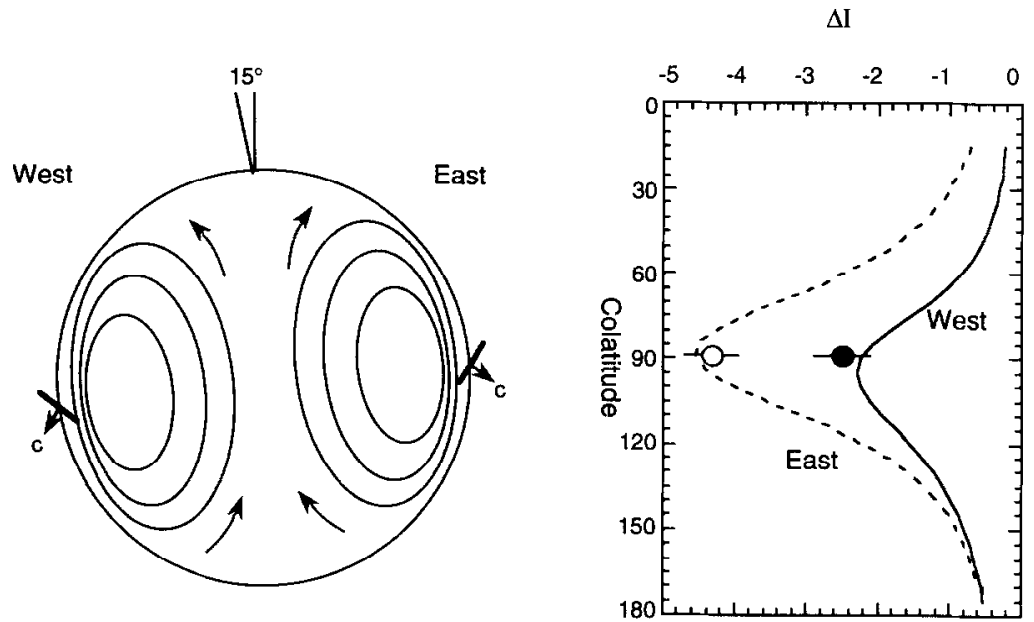

Fig. 2. The effects of a degree-two lateral heterogeneity in the preferred orientation of hcp iron in the inner core are illustrated here. The latitudes of the dipole moments are shifted plus or minus $15^{\circ}$ in the east and west hemispheres, and the resulting surface inclination anomalies are shown. For comparison the mean inclination anomalies for normal $(\bullet)$ and reverse $(O)$ polarity which form the basis of the polarity asymmetry interpretation are shown [10]. A sampling bias of this geometry which produces a better geographic distribution of normal polarity sites than of reverse polarity sites would average out the hemispheric differences to a greater extent in the normal polarity dataset than in the reverse polarity dataset. 
susceptibility. This may suggest a more uniformly magnetized inner core than expected from the convection geometry of Wenk et al. [31]. In this sense this model supports recent studies which indicate a stronger texturing throughout the inner core. Any evaluation of the magnitude of the induced moment, however, is hampered by the possible shielding effect of the outer core. In order for the effects of the induced magnetization to be observed at the surface, those effects must be amplified and transmitted by the dynamo process. The induced inner core moment, which changes more slowly than the field in the outer core, may provide a bias to the dynamo process which in turn affects the magnitude of the effect observed at the surface.

\section{Polarity asymmetry}

Whatever the process which produces the grain alignments in the inner core, it is very unlikely that this process is perfectly axisymmetric, and instead lateral variations are likely to exist. Misalignment of up to $35^{\circ}$ between the spin axis and the cylindrical symmetry axis of inner core anisotropy is consistent with the seismic data [24]. We can model the resulting inclination anomaly which would be observed at the Earth's surface if the axis of seismic anisotropy is tilted from the spin axis. The resulting inclination anomaly varies with longitude. In the modeled $\Delta I$ profiles shown in Fig. 2 we shifted the latitudes of the equatorial dipole moments by $\pm 15^{\circ}$ to illustrate how lateral variations could affect both the magnitude and shape of the inclination anomalies. These are shown for comparison with the average equatorial $\Delta I_{\mathrm{N}}$ and $\Delta I_{\mathrm{R}}$ values reported by Schneider and Kent [9]. In each hemisphere these anomalies will be the same during both normal and reverse polarity intervals, and therefore this type of lateral variation could not produce directly the observed polarity asymmetry. If the sampling of the time-averaged paleomagnetic field is geographically biased, however, the result would not be the perfect average of the two anomalies. For example, if the distributions of normal and re- verse polarity sites are not the same, a polarity bias would be expected simply because one polarity sampling would have averaged out the hemispheric asymmetry to a greater extent. In fact, in the recent studies of the time-averaged field there are consistently nearly twice the number of normal polarity sites than reverse polarity sites [9-13]. The normal polarity data also exhibit the smaller inclination anomaly, which is consistent with the greater averaging effect.

In order to examine the possibility of a sampling bias we calculated the $\Delta I$ anomalies for only those equatorial sites $\left( \pm 20^{\circ}\right.$ latitude) from Schneider and Kent's [11] database which exhibit both normal and reverse polarities at the same site. The deep-sea core dataset provides the broadest geographical distribution of equatorial sites where the polarity asymmetry effect is most likely to be observed. The $\Delta I$ values for this dataset also exhibit a significant polarity asymmetry: the normal polarity $\Delta I_{\mathrm{N}}\left(-2.25^{\circ}\right)$ is $2^{\circ}$ smaller than the reverse polarity $\Delta I_{\mathrm{R}}\left(-4.24^{\circ}\right)$.

The inclination anomalies plotted in Fig. 2, however, also show a difference between the range of inclination anomalies in the northern and southern hemispheres. The distribution of equatorial sites in Schneider and Kent's [11] database is not uniform in both hemispheres. The northern hemisphere sites, for example, are restricted to the western hemisphere between $164^{\circ} \mathrm{E}$ and $342^{\circ} \mathrm{E}$. These sites show a more pronounced asymmetry $\left(\Delta I_{\mathrm{N}}=-0.9, \Delta I_{\mathrm{R}}=-5.3\right)$. The southern hemisphere dual polarity sites are more evenly distributed from $58^{\circ} \mathrm{E}$ to $360^{\circ} \mathrm{E}$. These sites do not exhibit a polarity asymmetry, with $\Delta I_{\mathrm{N}}=-3.7^{\circ}$ and $\Delta I_{\mathrm{R}}=-3.2^{\circ}$. In other words the better longitudinally distributed dataset does not support the presence of a polarity asymmetry. It is important to note that the northern hemisphere dataset contains no sites between $8^{\circ} \mathrm{N}$ and $20^{\circ} \mathrm{N}$. Thus the difference between the two datasets may not only be due to the different longitudinal distribution but also to the difference in latitudinal distribution. Whereas these observations do not demonstrate a sampling bias effect they do however raise the possibility that the polarity asymmetry could result from a geographical sampling bias. 


\section{Polarity transition records}

Although more controversial than the anomalies in the time-averaged field, the recurrence of transitional VGP positions has also been interpreted as resulting from boundary conditions on the geodynamo. Several reversal records exhibit clustered transitional VGPs, which Hoffman [39] interpreted as resulting from flux patches in the core. Hoffman identified two preferred clusterings of VGPs which recur both within individual reversals and between reversals of different ages. These concentrations are located over Australia and South America and are correlated with features in the present day non-dipole field and P-wave anomalies in the lowermost mantle. Hoffman [39] suggested that the flux patches resulted from the influence of the core-mantle boundary, which caused the field to reorganize about these positions, resulting in an inclined dipolar state of the geomagnetic field. While it is clear that these clusterings are not present in all reversals $[17,18]$ recent data documenting the Brunhes-Matuyama reversal in Chile does add evidence supporting the Australian flux patch [40]. Additional support for the South American flux patch comes from records of the Nunivak reversals obtained from Fiji and Sicily (Fig. 3). The VGP paths from these records are similar to several others in that the VGPs persist over South America for approximately 2000-3000 yr. Although Van Hoof et al. [41] noted the probability that the Sicilian records had been heavily smoothed, they pointed out that the records are still consistent with Hoffman's South American flux patch biasing the VGP path over the Americas [41]. The clustering of VGPs in the records of both the upper and lower Nunivak from locations in opposite hemispheres is difficult to explain as a present-day field overprint and instead provides support for the influence of a flux patch beneath South America.

In order for VGPs from different sites to be
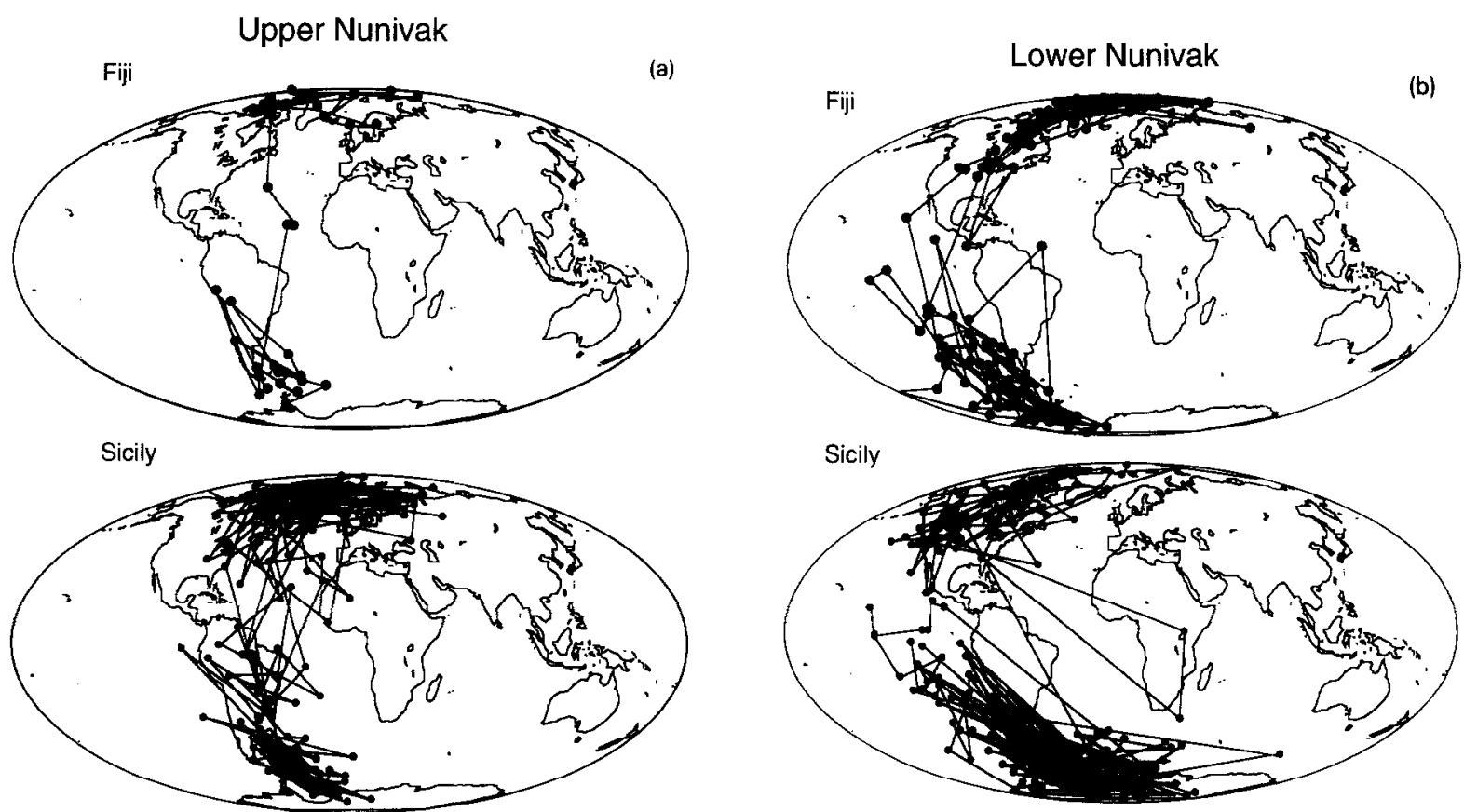

Fig. 3. The VGP paths during the upper and lower Nunivak polarity reversals recorded in Sicily [41] and Fiji. The records from opposite hemispheres yield remarkably similar VGP paths. In particular all four records exhibit a strong clustering of VGPs over South America which is difficult to interpret as a present-day field artifact. The location of these VGP concentrations is consistent with Hoffman's [19] proposed flux patches and supports the existence of recurring transitional VGP positions. 
clustered over the same area a nearly dipolar transitional field geometry is required at the surface. Although radial dipoles at the core-mantle boundary (CMB) are often used to explain largescale features of the geomagnetic field they are also subject to magnetic screening by the outer core. In order for a nearly dipolar field to result at the Earth's surface the inwardly directed field lines of the radial dipole must penetrate unaffected through the core to reach the surface on the far side of the core from the position of the radial dipole. Therefore the variations in the $\mathrm{CMB}$ would have to force a large-scale organization (throughout the outer core) of the transitional field in order to produce a nearly dipolar field at the surface. Even without shielding, a radial dipole at the CMB does not produce a dipolar field at the Earth's surface, and the resulting VGPs from a uniformly distributed global site distribution exhibit considerable scatter about a given mean position. A radial dipole at the top of the inner core produces a much more nearly dipolar field at the Earth's surface with a tighter grouping of transitional VGPs. For example, for a sampling of the field on a $20^{\circ}$ latitude by $30^{\circ}$ longitude grid the VGPs resulting from a CMB radial dipole group about a mean with an $\alpha_{95}$ of $8.1^{\circ}$ and $k=2.6$, whereas the VGPs resulting from an inner core dipole group about a mean with and an $\alpha_{95}$ of $2.4^{\circ}$ and $k=29.0$. But just as physical variations in the properties of the CMB must be transmitted through the outer core to cause a large-scale organization of the field, properties of the inner core must also affect the dynamo in order to produce the observed effects. The duration of the transitional VGP clusterings is of the same order as estimates of the time constant of magnetization of the inner core, and therefore it is worthwhile considering the effects of the inner core on the transitional field.

Hollerbach and Jones' [21,22] dynamo study suggests a reversal model in which the fluctuating outer core field becomes weak in the early stages of a random fluctuation that leads to a reversal. Because of the longer time constant, the inner core remains magnetized in the direction of the last, strong outer core field. While the outer core field is weak, the influence of the inner core field becomes greater, and a persisting inner core field may pin or serve as the seed field $\left(B_{0}\right)$ for the outer core field. As the outer core field grows in the opposite direction, it takes a few thousand years for the field to diffuse through and remagnetize the inner core. Thus the timing of the flux patches may be explained by the diffusive timescale of the inner core. The same dipole moments which produce the reversing quadrupole field (Fig. 1) could produce the southern hemisphere flux patches suggested by Hoffman [39]. A degree-two lateral variation in the easy direction of magnetization of the inner core, tilted approximately $15^{\circ}$ from the spin axis, which may explain the observed polarity asymmetry (Fig. 2), also might explain the different latitudes of Hoffman's two main flux patches.

After the outer core field has reversed the inner core is magnetized in the opposite direction. If the outer core field fluctuates to low values again the inner core field may produce VGPs clustered over a flux patch which is nearly antipodal to the one observed earlier during the reversal. In this way the reversing inner core field can produce the recurring transitional VGP positions observed within individual reversals and the antipodal character observed in several polarity transition paths $[15,16]$. If the inner core is assumed to corotate with the outer core and mantle this reversal scenario could also explain the recurrence of preferred VGP positions in different reversals.

\section{Discussion}

Although the intensity and geometry of the fields that diffuse into the inner core are unknown they are a function of the field in the outer core, and will be subject to the same symmetry arguments that apply to the dynamo field. Therefore it is difficult to explain the observed polarity asymmetry and the recurring transitional VGPs with simply the diffusing field alone. However, because of the different timescales at which the fields in the inner and outer core change it is possible that fields with geometries that ultimately give rise to the reversing quadrupole field 
could result if a mechanism could be found by which these geometries preferentially persist, or are amplified by the diffusion process. This could result if the fields described by the dipolar and quadrupolar families of solutions to the dynamo equations have different timescales of diffusion into the inner core. If the quadrupolar symmetry family diffuses at longer times than the dipolar symmetry family fields this might provide a possible explanation for the persisting quadrupole term. However, in order to explain the polarity asymmetry observed in the time-averaged paleomagnetic field, and the recurring transitional VGPs, a mechanism is required to produce a field in the inner core which is not precisely parallel to the field diffusing in from the outer core.

The model presented here provides a mechanism for explaining three different aspects of the Earth's magnetic field: The anomalies in the time-averaged field, polarity asymmetry, and the clustering of recurrent VGPs during polarity reversals. This model is highly speculative given the state of our knowledge of inner core conditions. However, it does provide a simple explanation for these different observations of field behavior. Given the uncertainties in the absolute magnitudes of physical properties of the inner core, evidence supporting the model is based primarily on arguments involving the timescales and geometries of changes in the geomagnetic field. In spite of the speculative nature of the model it is directly testable.

This model predicts that the observed polarity asymmetry is the result of a sampling bias; it may be tested by examining the inclination anomalies for normal and reverse polarity data from a uniform geographical distribution. If the inclination anomaly varies geographically, any dataset in which the data from one polarity are better distributed geographically will exhibit a polarity asymmetry. Most studies of the time-averaged field include nearly twice as many normal polarity sites as reverse polarity sites, consistent with the smaller $\Delta I$ anomaly for the normal polarity data. A re-examination of the time-averaged paleomagnetic field may provide important insights into the origin of the observed polarity asymmetry.
Central to this model is the magnitude of the magnetic susceptibility of the material that comprises the inner core. If the inner core is composed of hcp iron, room-temperature analogs of this material suggest that the susceptibility and the anisotropy of magnetic susceptibility is sufficient to produce an important effect at the top of the inner core. Unfortunately uncertainties in the absolute values of these quantities limit our calculations of the importance of these effects. Direct measurements of magnetic susceptibility at inner core conditions are hampered by the need to use metallic gaskets to provide the constraining pressure in diamond anvil experiments [Jeanloz, pers. commun., 1993]. Until the technological difficulties in making these measurements are overcome, first principles calculations may provide the best constraints on the possible range of susceptibilities and the anisotropy of magnetic susceptibility at inner core conditions [42].

Another difficulty with this model lies in how the effects of the inner core are transmitted through the electrically conducting and convecting outer core. It is clear that a magnetic field resulting from the magnetization of the inner core will not extend to the surface of the Earth without being affected by the dynamo process acting in the outer core. Hollerbach and Jones [21] point out that in their dynamo model the field lines that pass through the inner core also connect through to the mantle. Therefore the anisotropy of the inner core may have effects above the outer core. Although the mechanisms by which this might happen are unclear, in order for the mechanism proposed here to work the dynamo process must amplify the effects of inner core magnetization. There is little difference between invoking such a mechanism and calling for features at the CMB to cause large-scale organization of the field. Both involve transmission of field geometries through the dynamo. It may be that a persistent field at the top of the inner core provides a statistical bias to the dynamo. The inner core field would persist for periods corresponding to the magnetic diffusive timescale of the inner core which is significantly longer than the timescales of convection in the outer core. It is clear that we need to determine if such a 
statistical biasing is possible within dynamo theory and to determine what field strength would be required from the inner core.

\section{Conclusions}

We speculate that an inner core which is anisotropic in magnetic susceptibility can explain three different aspects of geomagnetic field behavior which have remained problematic: The time-averaged field anomaly, polarity asymmetry, and recurrent transitional VGPs. The inner core is elastically anisotropic [23-27], and if the elastic anisotropy results from a preferred orientation of a polycrystalline aggregate of hcp iron there is reason to believe that the inner core will also exhibit preferred directions of magnetization. Because of the finite electrical conductivity of the inner core its induced magnetization will change on the scale of a few thousand years, as opposed to the field of the outer core which changes on far shorter timescales. Although the inner core is well above the Curie temperature of iron the paramagnetic susceptibility of hcp iron is expected to be great enough that the magnetized inner core could provide a persistant statistical biasing field for the dynamo action occurring in the outer core. The simple model we present here serves to illustrate how an inner core with anisotropic electromagnetic properties could explain the reversing quadrupole field and the polarity asymmetry observed in the time-averaged paleomagnetic field, and recurring VGP positions during polarity reversals. Although this model remains highly speculative we do believe the success of such a simple model in explaining three different aspects of geomagnetic field behavior warrants further examination of the magnetic properties of iron at inner core conditions.

\section{Acknowledgements}

We thank Ken Hoffman and an anonymous reviewer for comments that improved this paper. C. Langereis kindly provided the data from the Nunivak polarity transitions from Sicily. This work was supported by the National Science Foundation under grant EAR-9305060 to L.P.S. and grant EAR-9304797 to B.M.C. L.P.S. was also supported by an Alexander von Humbolt Foundation Fellowship. [ $\boldsymbol{R} \boldsymbol{V}]$

\section{References}

[1] R.L. Wilson, Permanent aspects of the Earth's non-dipole magnetic field over upper Tertiary time, Geophys. J. R. Astron. Soc. 19, 417-437, 1970.

[2] R.L. Wilson, Paleomagnetic differences between normal and reversed field sources and the problem of far-sided and right-handed pole positions, Geophys. J. R. Astron. Soc. 28, 295-304, 1972.

[3] D.H. Coupland and R. van der Voo, Long-term nondipole components in the geomagnetic field during the last 130 m.y., J. Geophys. Res. 85, 3529-3548, 1980.

[4] R.T. Merrill and M.W. McElhinny, Anomalies in the time-averaged paleomagnetic field and their implications for the lower mantle, Rev. Geophys. 15, 309-323, 1977.

[5] R.T. Merrill, M.W. McElhinny and D.J. Stevenson, Evidence for long term asymmetries in the Earth's magnetic field and possible implications for dynamo theories, Phys. Earth Planet. Inter. 20, 75-82, 1979.

[6] R.T. Merrill, P.L. McFadden and M.W. McElhinny, Paleomagnetic tomography of the core-mantle boundary, Phys. Earth Planet. Inter. 64, 87-101, 1990.

[7] R.A. Livermore, F.J. Vine and A.G. Smith, Plate motions and the geomagnetic field I: Quaternary and late Tertiary, Geophys. J. R. Astron. Soc. 73, 153-171, 1983.

[8] R.A. Livermore, F.J. Vine and A.G. Smith, Plate motions and the geomagnetic field II: Jurassic to Tertiary, Geophys. J. R. Astron. Soc. 73, 153-171, 1984.

[9] D.A. Schneider and D.V. Kent, The paleomagnetic field from deep-sea sediments: Axial symmetry and polarity asymmetry, Science 242, 252-256, 1988.

[10] D.A. Schneider and D.V. Kent, Inclination anomalies from Indian Ocean sediments and the possibility of a standing nondipole field, J. Geophys. Res. 93, 11621$11630,1988$.

[11] D.A. Schneider and D.V. Kent, The time-averaged paleomagnetic field, Revs. Geophys. 28, 71-96, 1990.

[12] C. Johnson and C. Constable, Palaeosecular variation recorded by lava flows over the past $5 \mathrm{Ma}$, Philos. Trans. R. Soc. London, in press.

[13] X. Quidelleur, J.-P. Valet, V. Courtillot and G. Hulot, Long-term geometry of the geomagnetic field for the last five million years: An updated secular variation data base, Geophys. Res. Lett. 21, 1639-1642, 1994.

[14] C. Laj, A. Mazaud, R. Weeks, M. Fuller and E. Herrero-Brevera, Geomagnetic reversal paths, Nature 351, 447, 1991.

[15] B.M. Clement, Geographical distribution of transitional VGPs: Evidence for non-zonal equatorial symmetry dur- 
ing the Matuyama-Brunhes geomagnetic reversal, Earth Planet. Sci. Lett. 104, 48-58, 1991.

[16] B.M. Clement and D.G. Martinson, A quantitative comparison of two paleomagnetic records of the Cobb Mountain Subchronozone from North Atlantic deep-sea sediments, J. Geophys. Res. 97, 1735-1752, 1992.

[17] M. Prevot and P. Camps, Absence of preferred longitude sectors for poles from volcanic records of geomagnetic polarity reversals, Nature 366, 53-56, 1993.

[18] J.-P. Valet, P. Tucholka, V. Courtillot and L. Meynadier, Paleomagnetic constraints on the geometry of the geomagnetic field during reversals, Nature 356, 400-407, 1992.

[19] K.A. Hoffman, Long-lived transitional states of the geomagnetic field and the two dynamo familes, Nature 354 , 273-277, 1991.

[20] A.M. Dziewonski and J.H. Woodhouse, Global images of the earth's interior, Science 236, 37-48, 1987.

[21] R. Hollerbach and C.A. Jones, Influence of the Earth's inner core on geomagnetic fluctuations and reversals, Nature 365, 541-543, 1993.

[22] R. Hollerbach and C.A. Jones, A geodynamo model incorporating a finitely conducing inner core, Phys. Earth Planet. Inter. 75, 317-327, 1993.

[23] A. Morelli, A.M. Dziewonski and J.H. Woodhouse, Anisotropy of the inner core inferred from PKIKP travel times, Geophys. Res. Lett. 13, 1545-1548, 1986.

[24] K.C. Creager, Anisotropy of the inner core from differential travel times of the phases PKP and PKIKP, Nature 356, 309-314, 1992.

[25] X. Song and D.V. Helmberger, Anisotropy of earth's inner core, Geophys. Res. Lett. 20, 2591-2594, 1993.

[26] J.H. Woodhouse, D. Giardini and X.-D. Li, Evidence for inner core anisotropy from free oscillations, Geophys. Res. Lett. 13, 1549-1552, 1986.

[27] J. Tromp, Support for anisotropy of the earth's inner core from free oscillations, Nature 366, 678-681, 1993.

[28] L. Stixrude and R.E. Cohen, High pressure and temperature elasticity of iron: Implications for the inner core, Science, in press.

[29] R. Jeanloz and H. Wenk, Convection and anisotropy of the inner core, Geophys. Res. Lett. 15, 72-75, 1988.
[30] S. Karato, Inner core anisotropy due to the magnetic field induced preferred orientation of iron, Science 262, 1708-1711, 1993.

[31] H.R. Wenk, T. Takeshita, R. Jeanloz and G.C. Johnson, Development of texture and elastic anisotropy during deformation of hcp metals, Geophys. Res. Lett. 15, 76-79, 1988.

[32] K.A. Verosub, Alternative to the geomagnetic self-reversing dynamo, Nature 253, 707-708, 1975.

[33] C.S. Wang, B.M. Klein and H. Krakauer, Theory of magnetic and structural ordering in iron, Phys. Rev. Lett. $54,1852-1855,1985$.

[34] L. Stixrude, R.E. Cohen and D.J. Singh, Iron at high pressure: Linearized augmented plane wave calculations in the generalized gradient approximation, Phys. Rev. B, in press.

[35] C. Kittel, Introduction to Solid State Physics, 5th ed., Chap. 14, Wiley, New York, 1976.

[36] I. Bakonyi, H. Ebert and A.I. Liechtenstein, Electronic structure and magnetic susceptibility of the different structural modifications of $\mathrm{Ti}, \mathrm{Zr}$, and $\mathrm{Hf}$ metals, Phys. Rev. B 48, 7841-7849, 1993.

[37] E.W. Collings and J.C. Ho, Magnetic susceptibility and low-temperature specific heat studies of $\mathrm{Ti}, \mathrm{Zr}$, and $\mathrm{Hf}$, Phys. Rev. B 4, 349-356, 1971.

[38] K. Zhang and D.R. Fearn, How strong is the invisible component of the magnetic field in the Earth's core?, Geophys. Res. Lett. 20, 2083-2086, 1993.

[39] K.A. Hoffman, Dipolar reversal states of the geomagnetic field and core-mantle dynamics, Nature 359, 789794, 1992.

[40] L. Krown, J. Pickens and B. Singer, Matuyama-Brunhes transition recorded in lava flows of the Chilean Andes: Evidence for dipolar fields during reversals, Geology 22 , 289-384, 1994.

[41] A.A.M. van Hoof B.J. van Os and C.G. Langereis, The upper and lower Nunivak sedimentary geomagnetic transition records from Southern Sicily, Phys. Earth Planet. Inter. 77, 297-314, 1993.

[42] D.J. Singh and J. Ashkenazi, Magnetism with generalized-gradient-appproximation density functionals, Phys. Rev. B-Condensed Matter 46, 11570-11577, 1992. 